\title{
Nanoparticles in Cancer Imaging and Therapy
}

\author{
Leon Smith, ${ }^{1}$ Zdenka Kuncic, ${ }^{1}$ Kostya (Ken) Ostrikov, ${ }^{1,2}$ and Shailesh Kumar ${ }^{2}$ \\ ${ }^{1}$ Institute of Medical Physics, School of Physics, The University of Sydney, Sydney, N.S.W 2006, Australia \\ ${ }^{2}$ Plasma Nanoscience Centre Australia (PNCA), CSIRO Materials Science and Engineering, P.O. Box 218, Lindfield, \\ N.S.W 2070, Australia \\ Correspondence should be addressed to Zdenka Kuncic, zdenka.kuncic@sydney.edu.au
}

Received 10 February 2012; Accepted 3 March 2012

Academic Editor: Krasimir Vasilev

Copyright () 2012 Leon Smith et al. This is an open access article distributed under the Creative Commons Attribution License, which permits unrestricted use, distribution, and reproduction in any medium, provided the original work is properly cited.

Nanoparticle contrast agents offer the potential to significantly improve existing methods of cancer diagnosis and treatment. Advantages include biocompatibility, selective accumulation in tumor cells, and reduced toxicity. Considerable research is underway into the use of nanoparticles as enhancement agents for radiation therapy and photodynamic therapy, where they may be used to deliver treatment agents, produce localized enhancements in radiation dose and selectively target tumor cells for localized damage. This paper reviews the current status of nanoparticles for cancer treatment and presents preliminary results of a pilot study investigating titanium dioxide nanoparticles for dual-mode enhancement of computed tomography (CT) imaging and kilovoltage radiation therapy. Although titanium dioxide produced noticeable image contrast enhancement in the CT scans, more sensitive detectors are needed to determine whether the nanoparticles can also produce localized dose enhancement for targeted radiation therapy.

\section{Introduction}

Contrast agents are now standard practice in the field of medical imaging, where they are used to enhance image contrast and improve the visibility of features that would otherwise be difficult to detect [1]. Nanoparticle agents continue to receive considerable attention in this field for their potential as contrast agents [2], offering the advantage of greater biocompatibility and reduced toxicity compared to more conventional chemical agents [3]. Present nanoparticles under development for this role include gold nanoparticles for X-ray contrast enhancement [3], magnetic nanoparticles for MRI enhancement [4], and even hybrid nanoparticles containing iron oxide and gold in a polymer coating, which serve as contrast agents for both CT and MRI [5].

In addition to imaging, nanoparticle agents are also being investigated for potential applications to cancer therapy [6-8], where they offer similar advantages over other contrast agents as they do in imaging, coupled with the potential for designing nanoparticles that can selectively accumulate in cancer cells, providing "targeted" treatments that may not be possible with conventional techniques [7]. Indeed, nanoparticles have proven their use as a general platform for targeted drug delivery, owing to the ability for tethered functionality such as an antibody agent or quantum dot fluorescent reporter to enhance delivery of chemotherapeutic agents [9] (Figure 1). There is now also growing interest in the use of nanoparticles as enhancement agents in cancer radiation therapy and photodynamic therapy to improve patient outcomes and reduce side effects. As an increasing number of cancer patients are treated with radiotherapy, there is potential for a much wider impact of nanoparticleenhanced radiotherapy.

In this paper, we review the current status of nanoparticle enhancement agents for radiation therapy and photodynamic therapy. In addition, we present preliminary results for a pilot study investigating the use of titanium dioxide nanoparticles as possible dual-mode enhancement agents for computed tomography (CT) imaging and radiation therapy.

\section{Contrast-Enhanced Radiation Therapy with Nanoparticles}

There has been considerable investigation into the potential of traditional imaging contrast agents, such as iodine, for 


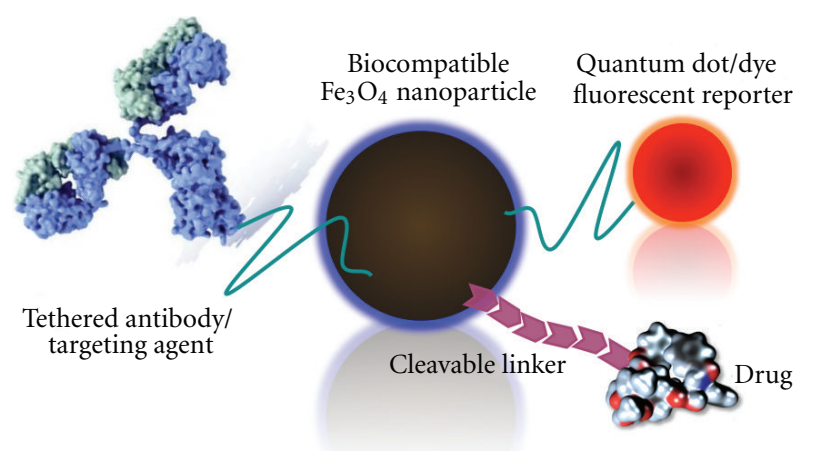

FIGURE 1: Schematic of an iron-based nanoparticle showing multifunctionality for image contrast and targeted drug delivery. (With kind permission from A. L. Yuen, School of Chemistry, University of Sydney.)

applications to radiation therapy cancer treatment [10-13]. During radiation therapy, ionising radiation is delivered to the tumor, causing damage to the tumor cells' DNA and other biological molecules, resulting in the death of the cell. Experiments on mice bearing Ehrlich ascites carcinoma treated with iodine contrast agents have shown complete regression in up to $80 \%$ of mice tested [10].

An inherent limitation of radiation therapy is the unavoidable radiation dose delivered to healthy tissue surrounding the tumor being treated. For some tumors, this has limited the use of radiotherapy as the necessary dose to destroy the tumor will also cause unacceptable damage to nearby healthy tissue [14]. Contrast-enhanced radiotherapy (CERT) [6] involves the administration of high- $Z$ (i.e., high atomic number) contrast agents to the target volume and irradiation with kilovoltage X-rays. As high- $Z$ materials have different absorption properties from surrounding tissue, their presence can modify the distribution in absorbed dose; in particular, a localized dose enhancement can be obtained in regions where the contrast agent is present without affecting healthy tissue $[11,12]$. As this difference in photoabsorption properties is most pronounced at kilovoltage energies $[6,15]$, kilovoltage X-rays are generally considered to be the optimum energy range for CERT [12], but several studies have also investigated contrast enhancement using $\mathrm{X}$-rays in the megavoltage energy range produced by medical linear accelerators $[16,17]$.

In recent years, studies into potential radiotherapy contrast agents have often focused on gold nanoparticles [16-21]. Gold nanoparticles have a number of useful properties that make them highly attractive contrast agents for cancer therapy, particularly their high $Z$ and biocompatibility $[21,22]$. Monte Carlo simulations of interactions between the radiation treatment beam and individual nanoparticles indicate that the presence of a gold nanoparticle can increase secondary electron production from a $50 \mathrm{kV}$ X-ray beam by up to a factor of 2000 compared to a similar volume of water [23] (Figure 2). Nanoparticles smaller than $100 \mathrm{~nm}$ can also cross human cell membranes and preferentially accumulate in cancer cells [24]. Recent studies have found that gold nanoparticles in conjunction with kilovoltage radiotherapy can increase the one-year survival rate of mice

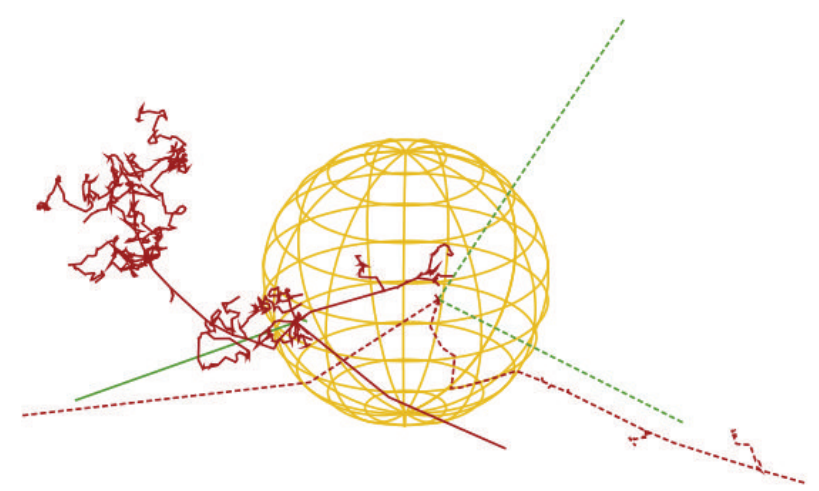

Figure 2: Monte Carlo visualization of the interaction of a lowenergy photon (green lines) with a $20 \mathrm{~nm}$ gold nanoparticle (yellow sphere), showing electron tracks (red) associated with ionization events. Solid and dashed lines correspond to events outside and inside the nanoparticle, respectively. (Reprinted by permission from Macmillan Publishers Ltd: Scientific Reports [22], copyright 2011.)

with EMT-6 mammary tumors to $86 \%$, compared to $20 \%$ with radiotherapy alone [19].

It has been observed that gold nanoparticles appear to cause clinically relevant increases in cell killing rates at concentrations well below those predicted by Monte Carlo simulations $[22,25]$. Although the simulations predict that cell killing effects should become noticeable only at nanoparticle concentrations on the order of $1 \%$, they have been observed at concentrations orders of lower magnitude [22]. It has been speculated that this discrepancy is due to the Monte Carlo averaging of the effects of the nanoparticles over macroscopic volumes, thereby ignoring nanoscale inheterogeneities in dose distribution in the vicinity of each nanoparticle $[22,23]$. Simulating these inheterogeneities and their corresponding biological effects predicts cell killing at concentrations closer to those reported in experimental studies [22].

An additional factor that is relevant to the effects of nanoparticles on cell killing is their radiosensitization properties. There is increasing evidence that gold nanoparticles, while biocompatible, are not necessarily biologically inert [25], which influences their effects on cells during cancer therapy. Gold nanoparticles have also demonstrated the capacity to form radiosensitized DNA molecules in cells they affect, making them more susceptible to radiation whilst simultaneously increasing localised dose near the target [21]. One consequence of these biological effects is that gold nanoparticles appear to demonstrate considerably greater cell killing effects in some cell lines as compared to others [25], which should be taken into consideration in future studies.

\section{Nanoparticles in Photodynamic Cancer Therapy}

Photodynamic therapy (PDT) is a treatment modality which involves administering a photosensitive agent and 
illuminating the target area to activate the agent $[26,27]$. In cancer therapy, existing PDT agents react upon photostimulation to produce cytotoxic oxygen species, killing the target cell $[27,28]$. Some of the advantages of PDT include its localized effects, reduced cost, and the fact that it is largely an outpatient therapy [27]. Interestingly, it can also induce immune responses even against tumors that are not particularly immunogenic [7]. One of its primary limitations, however, is the limited penetration depth of visible, infrared, and UV radiation into the patient [27-29] and the lack of effective dosimetry techniques for PDT, making it difficult for dose distributions in the treatment volume to be accurately measured [30].

As of 2010, only three classes of photosensitisers for PDT had entered clinical use for cancer therapy $[28,30]$, all of them chemical products rather than nanoparticles. However, a myriad of nanoparticle PDT agents are presented in various stages of development and show promise against a wide range of cancers. These nanoparticles can be separated into "passive" nanoparticles, which carry photosensitive agents, and "active" particles, which are themselves involved in the photostimulation process [7].

3.1. Passive Nanoparticles. A wide range of materials are in use for passive nanoparticles, including gold [31], polyacrylamide [32], silica [33], and biodegradable polymers [7]. One of their primary advantages over unencapsulated photosensitizers is their ability to preferentially accumulate in target cells $[7,33]$, which is of particular use given that most photosensitizers described so far in the literature have a low solubility in water and tend to accumulate in blood cells rather than tumors. This allows similar therapeutic effects to be achieved with lower doses of conventional photosensitizers such as hypericin, limiting their side effects [34]. Encapsulating potentially toxic photosensitizers inside nanoparticles is another possibility, enabling their use in patients when this might not otherwise be possible [7]. One issue that must be considered in passive photodynamic nanoparticles is the optimum drug concentration within the nanoparticle to achieve maximum therapeutic effect, as excessive drug loading can reduce the overall effect of the nanoparticles [34].

There is increasing presence in the literature of biodegradable passive nanoparticles for photodynamic therapy, typically made from polylactic acid or poly(lactic-coglycolic acid) [7]. These nanoparticles can selectively deliver photosensitizers to target cells and then break down inside the cell, releasing the photosensitizer into target cells. This approach has seen impressive results in mouse models; in a study involving the administration of the photosensitizer hexadecafluoro zinc phthalocyanine to EMT-6 mammary tumors in mice, encapsulation of the photosensitizer in polylactic acid nanoparticles resulted in $100 \%$ of tested mice achieving tumor regression, compared with only $60 \%$ of mice administered the free drug [35]. Nanoparticle encapsulation of Indocyanine green, recently evaluated as a possible photosensitizer, was observed to increase organ deposition to the photosensitizer to levels $2-8$ times that for the free drug [36]. Other photosensitizers which have observed beneficial results from encapsulation in biodegradable nanoparticles include hypericin [34] and zinc (II) phthalocyanine [37].

3.2. Active Nanoparticles. A rare example of nanoparticles that can act as a photosensitizer on their own are titanium dioxide $\left(\mathrm{TiO}_{2}\right)$ nanoparticles, which are receiving considerable attention as $\mathrm{TiO}_{2}$ is a known photocatalyst and reacts with water to produce oxidizing free radicals when exposed to UV light [38], which can result in localized damage to nearby cells [38-40]. Recent in vitro studies on glioma cells have demonstrated the potential of such nanoparticles for photodynamic therapy [39]. A similar effect has recently been produced in $\mathrm{TiO}_{2}$ nanoparticles with ultrasonic stimulation, which is able to kill nanoparticle-impregnated glioma cells when exposed to ultrasound in a similar manner to UVstimulated nanoparticles [29]. $\mathrm{TiO}_{2}$ nanoparticles are also essentially non toxic [41-43] and hence show considerable promise as cancer therapy agents. Other nanoparticles that have recently been shown to demonstrate photosensitizer effects include porous silicon and carbon-60 buckyballs $[8,44]$.

Other active nanoparticles generally serve to enhance the photodynamic process and are still used in conjunction with a photosensitizer. The aim of these nanoparticles is usually to generate light at frequencies useful for photodynamic therapy in regions deeper inside the patient, sidestepping the issue of the limited penetration hampering photodynamic therapy at non superficial depths. The majority of studies in this area has focused on "upconverting" nanoparticles, which convert low-frequency near infrared radiation (NIR) into frequencies usable for existing photosensitizers [7]. As well as penetrating further into the patient, NIR is also less damaging to the patient.

The most popular upconverting material for such nanoparticles at present is sodium yttrium fluoride doped with erbium and yttrium ions with a poly(ethylene imine) coat, forming PEI/NaYF $4: \mathrm{Yb}^{3+}, \mathrm{E}^{\mathrm{r} 3+}$ nanoparticles $[7,27]$. Proof-of-concept studies in rats have confirmed that these upconverting nanoparticles can activate zinc phthalocyanine photosensitisers in rats, with resultant therapeutic effects, upon stimulation with NIR radiation well outside the absorption spectrum of the photosensitizer [27]. Upconverting nanoparticles, their photosensitizer, and a biocompatible layer may be combined into "composite nanoparticles," which can produce millimolar quantities of cytotoxic radicals upon illumination with NIR [45]. The upconverting phosophor used in these nanoparticles may also be activated with X-ray radiation, which would completely eliminate the depth limitations of photodynamic therapy, at the expense of increased healthy tissue damage [45].

\section{Nanoparticles for Dual-Mode Imaging and Therapy?}

One area that remains relatively unexplored is the concept of agents for dual-mode image contrast and therapy enhancement. The concept of dual-mode enhancement agents has 
been tested using traditional non-nanoparticle image contrast agents, such as iodine, for radiotherapy enhancement applications [11-13]. As iodine is routinely used as an image contrast agent for CT scans [46], a conventional CT scanner could in principle be used to simultaneously monitor tumor progression and iodine uptake.

For nanoparticle-based enhancement agents, progress remains limited, although initial studies combining MRI enhancement and photodynamic therapy have recently appeared in the literature $[47,48]$. If nanoparticles for radiotherapy using ionizing radiation were developed, however, it would be desirable to detect them on CT scanners instead. CT scanners are the standard imaging technique for diagnosis and treatment planning in virtually all cancer centres [49] and are increasingly replacing traditional simulator units for radiotherapy treatment planning. It is even possible to modify a conventional CT scanner to deliver therapeutic X-ray doses using iodine CERT $[12,50]$. This treatment modality produces dose distributions similar to those obtained with $10 \mathrm{MV}$ megavoltage therapy [12] and enables combined imaging, simulation, and treatment with the same machine. A similar option for more sophisticated contrast agents such as $\mathrm{TiO}_{2}$ would be highly desirable and deserves investigation.

4.1. A Pilot Study with $\mathrm{TiO}_{2}$ Nanoparticles. We conducted a pilot study investigating the potential of $\mathrm{TiO}_{2}$ nanoparticles as a potential contrast agent for dual-mode imaging and therapy. We measured the effects of each of these agents on dose delivered by a kilovoltage X-ray therapy unit and imaged nanoparticle-impregnated volumes with a clinical CT scanner to determine their image contrast properties.

4.1.1. Methodology. In this study, rutile phase $\mathrm{TiO}_{2}$ nanoparticles, with a diameter of $2-3 \mathrm{~nm}$, were used. The concentration of these solutions ranged from 0 to $0.231 \mathrm{~g} / \mathrm{mL}$. The nanoparticles were fabricated using a DC magnetronassisted sputtering deposition system. Ultra-high-purity argon and oxygen (both of purity greater than 99.99\%) were used as the sputtering and reactive gases, respectively. The deposition process was carried out on ultrasonically cleaned silicon wafers.

To evaluate their imaging capabilities, the nanoparticle solutions were placed in a cylindrical plastic vial at a depth of $1.5 \mathrm{~cm}$. These vials were imaged using a Toshiba Aquilion wide-bore CT scanner. The CT numbers of each vial in Hounsfield Units (the standard units used in CT scans [45]) of each vial were plotted against concentration and a linear fit was produced.

To evaluate their dose enhancement capabilities, the solutions were irradiated on a Pantak Therapax DXT $300 \mathrm{kVp}$ $\mathrm{X}$-ray unit. The solutions were placed in plastic containers on the surface of an RMI solid-water phantom, such that the nanoparticle solution had a thickness of $0.5 \mathrm{~cm}$. Pieces of radiation-sensitive EBT2 radiochromic film were placed on the surface of the solution, such that the active layer was facing the radiation source. The film was then irradiated with a dose of $1.00 \mathrm{~Gy}$ at the surface. The dose recorded by each

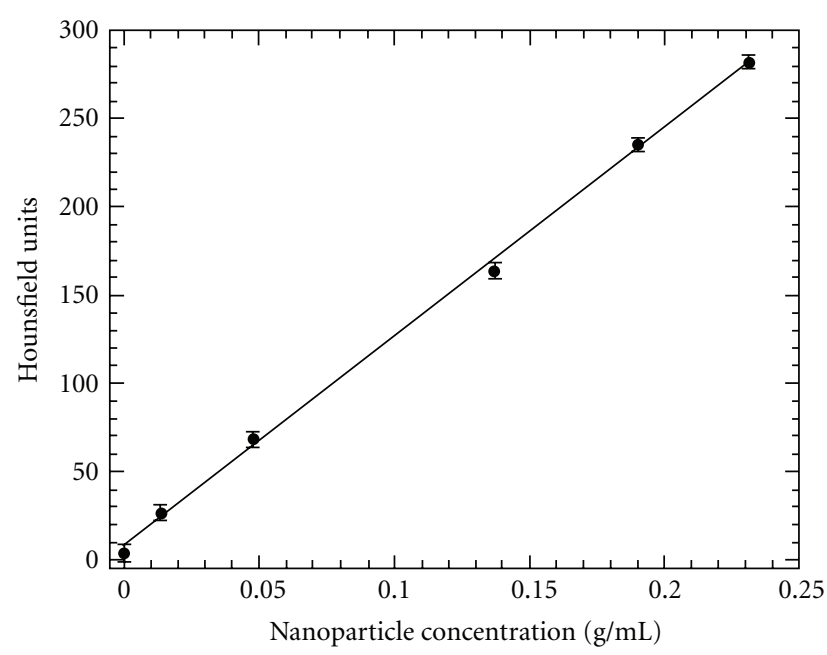

FIGURE 3: CT numbers of titanium dioxide nanoparticle solutions as a function of concentration (symbols, with error bars) and a linear best fit.

film piece was plotted against nanoparticle concentration to determine if the presence of the nanoparticles produced a dose enhancement. The dose values were normalized with respect to the dose recorded for the solution containing no nanoparticles.

4.1.2. Results. The CT numbers of the scanned nanoparticle concentrations are shown in Figure 3. A sample scan is shown in Figure 4. The CT numbers of the nanoparticle solutions increase approximately linearly with nanoparticle concentration, ranging from $0 \pm 5 \mathrm{HU}$ (no nanoparticles) to $281.5 \pm 4 \mathrm{HU}(0.231 \mathrm{~g} / \mathrm{mL})$. The uncertainty in each measurement (given by the standard deviation of the Hounsfield unit measurements) is within $5 \mathrm{HU}$ for each measurement. A nanoparticle concentration of $0.015 \mathrm{~g} / \mathrm{mL}$ gives a reading of $26.6 \pm 4 \mathrm{HU}$, which is greater than the typical noise value of about $5 \mathrm{HU}$ reported on most CT scanners [46]. These results indicate that even at low concentrations, the presence of $\mathrm{TiO}_{2}$ nanoparticles produces detectable changes in the CT numbers of scanned media which are detectable on a conventional medical CT scanner.

The surface doses recorded by the EBT2 film pieces as a function of nanoparticle concentration are shown in Figures 5 and 6. For both energies, no enhancement effects in surface dose are observed. In both cases, the dose measured in the solution containing no nanoparticles is the highest measured. The dose measured at $50 \mathrm{kVp}$ decays approximately monotonically with nanoparticle concentration. For the $125 \mathrm{kVp}$ beam energy, the fluctuations observed in the dose measurements for all nanoparticle concentrations are within the $4 \%$ calculated uncertainty. None of these values are more than $97 \%$ of the dose measured for the vial containing no nanoparticles. It is possible that the reduction in surface dose at higher nanoparticle concentrations is due to absorption of backscattered radiation near the surface by the nanoparticle solutions, with no corresponding dose enhancement to compensate for this. As such, it can be 


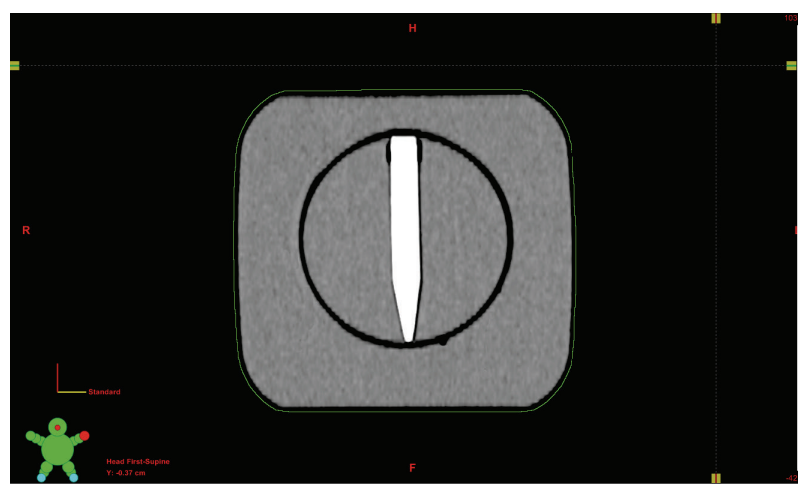

Figure 4: Sample coronal CT scan of a nanoparticle-containing vial. The nanoparticle solution (of high attenuation) appears as white, whereas the plastic frame containing the vial (of low attenuation) appears black.

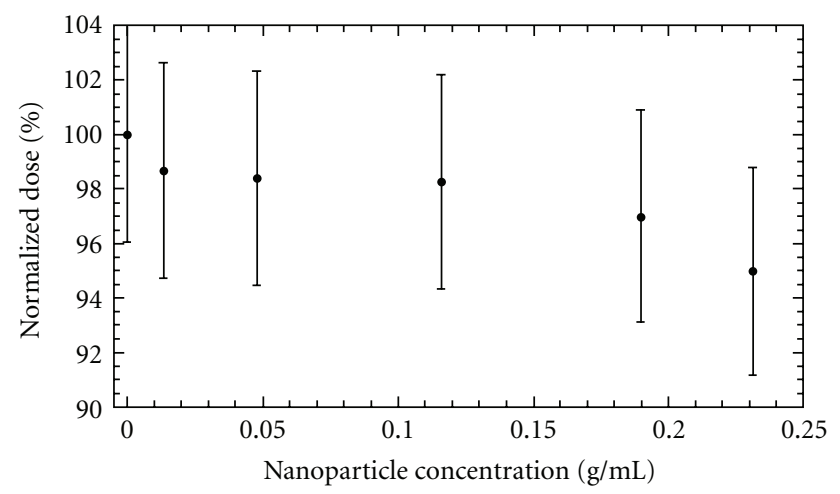

Figure 5: Normalized surface dose recorded for the $50 \mathrm{kVp}$ radiation beam as a function of nanoparticle concentration.

concluded that a therapeutic enhancement in surface dose is not observable using $\mathrm{TiO}_{2}$ nanoparticles of the phase and concentrations investigated in this study, to within the sensitivity limits of the film dosimetry technique that was used.

\section{Conclusion}

Nanoparticles offer enormous potential for improving cancer imaging and treatment. With demonstrated success in existing diagnostic imaging and drug delivery techniques, there is now growing interest in the potential applications to radiation therapy and photodynamic therapy. Several classes of nanoparticles are presently under development for both of these techniques and are likely to enable significant improvements to the efficacy of existing cancer therapy regimes in the foreseeable future. We reviewed the current status of nanoparticle agents for cancer therapy. Preliminary results for titanium dioxide nanoparticles as potential dualmode imaging and therapy enhancement agents indicate they are a promising candidate for image contrast in computed tomography. However, more comprehensive studies are needed, with more sensitive detection techniques, to

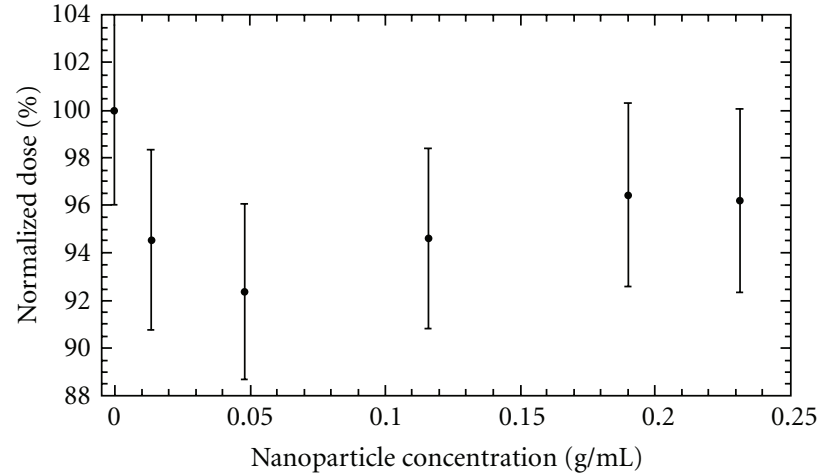

Figure 6: Normalized surface dose recorded for the $125 \mathrm{kVp}$ radiation beam as a function of nanoparticle concentration.

determine whether they may also serve to enhance radiation dose for targeted cancer radiotherapy applications.

\section{Acknowledgments}

L. Smith acknowledges support from a CSIRO Scholarship. The authors wish to acknowledge Royal Prince Alfred Hospital, Sydney, Australia, for access to clinical facilities and also wish to thank A. L. Yuen at the School of Chemistry, University of Sydney, for providing Figure 1. This work is supported by the Australian Research Council (ARC) and CSIRO's OCE Science Leadership Program.

\section{References}

[1] P. Suetens, Fundamentals of Medical Imaging, Cambridge University Press, New York, NY, USA, 2nd edition, 2009.

[2] P. Jackson, S. Periasamy, V. Bansal, and M. Geso, "Evaluation of the effects of gold nanoparticle shape and size on contrast enhancement in radiological imaging," Australasian Physical and Engineering Sciences in Medicine, vol. 34, no. 2, pp. 243249, 2011.

[3] J. F. Hainfeld, D. N. Slatkin, T. M. Focella, and H. M. Smilowitz, "Gold nanoparticles: a new X-ray contrast agent," British Journal of Radiology, vol. 79, no. 939, pp. 248-253, 2006.

[4] C. Fang and M. Zhang, "Multifunctional magnetic nanoparticles for medical imaging applications," Journal of Materials Chemistry, vol. 19, no. 35, pp. 6258-6266, 2009.

[5] D. Kim, M. K. Yu, T. S. Lee, J. J. Park, Y. Y. Jeong, and S. Jon, "Amphiphilic polymer-coated hybrid nanoparticles as CT/MRI dual contrast agents," Nanotechnology, vol. 22, no. 15, Article ID 155101, 2011.

[6] H. M. Garnica-Garza, "Contrast-enhanced radiotherapy: feasibility and characteristics of the physical absorbed dose distribution for deep-seated tumors," Physics in Medicine and Biology, vol. 54, no. 18, pp. 5411-5425, 2009.

[7] D. K. Chatterjee, L. S. Fong, and Y. Zhang, "Nanoparticles in photodynamic therapy: an emerging paradigm," Advanced Drug Delivery Reviews, vol. 60, no. 15, pp. 1627-1637, 2008.

[8] B. C. Wilson and M. S. Patterson, "The physics, biophysics and technology of photodynamic therapy," Physics in Medicine and Biology, vol. 53, no. 9, pp. R61-R109, 2008. 
[9] A. K. L. Yuen, G. A. Hutton, A. F. Masters, and T. Maschmeyer, "The interplay of catechol ligands with nanoparticulate iron oxides," Dalton Transactions, vol. 41, no. 9, pp. 2545-2559, 2012.

[10] R. S. Mello, H. Callison, J. Winter, A. R. Kagan, and A. Norman, "Radiation dose enhancement in tumors with iodine," Medical Physics, vol. 10, no. 1, pp. 75-78, 1983.

[11] A. V. Mesa, A. Norman, T. D. Solberg, J. J. Demarco, and J. B. Smathers, "Dose distributions using kilovoltage X-rays and dose enhancement from iodine contrast agents," Physics in Medicine and Biology, vol. 44, no. 8, pp. 1955-1968, 1999.

[12] J. H. Rose, A. Norman, and M. Ingram, "First experience with radiation therapy of small brain tumors delivered by a computerized tomography scanner," International Journal of Radiation Oncology Biology Physics, vol. 30, pp. 1127-1132, 1994.

[13] F. Verhaegen, B. Reniers, F. Deblois, S. Devic, J. Seuntjens, and D. Hristov, "Dosimetric and microdosimetric study of contrast-enhanced radiotherapy with kilovolt x-rays," Physics in Medicine and Biology, vol. 50, no. 15, pp. 3555-3569, 2005.

[14] A. R. Kagan, R. J. Steckel, P. Cancilla, G. Juillard, and T. Patin, "The pathogenesis of brain necrosis: time and dose parameters," International Journal of Radiation Oncology Biology Physics, vol. 1, no. 7-8, pp. 729-732, 1976.

[15] S. J. McMahon, M. H. Mendenhall, S. Jain, and F. Currell, "Radiotherapy in the presence of contrast agents: a general figure of merit and its application to gold nanoparticles," Physics in Medicine and Biology, vol. 53, no. 20, pp. 5635-5651, 2008.

[16] R. I. Berbecoa, W. Ngwaa, and M. Makrigiorgosa, "Localized dose enhancement to tumor blood vessel endothelial cells via targeted gold nanoparticles: new potential for external beam radiotherapy," International Journal of Radiation Oncology Biology Physics, vol. 78, pp. S649-S650, 2010.

[17] D. B. Chithrani, S. Jelveh, F. Jalali et al., "Gold nanoparticles as radiation sensitizers in cancer therapy," Radiation Research, vol. 173, no. 6, pp. 719-728, 2010.

[18] J. F. Hainfeld, F. A. Dilmanian, D. N. Slatkin, and H. M. Smilowitz, "Radiotherapy enhancement with gold nanoparticles," The Journal of Pharmacy and Pharmacology, vol. 60, no. 8, pp. 977-985, 2008.

[19] J. F. Hainfeld, D. N. Slatkin, and H. M. Smilowitz, "The use of gold nanoparticles to enhance radiotherapy in mice," Physics in Medicine and Biology, vol. 49, no. 18, pp. N309-N315, 2004.

[20] Y. Zheng, P. Cloutier, D. J. Hunting, and L. Sanche, "Radiosensitization by gold nanoparticles: comparison of DNA damage induced by low and high-energy electrons," Journal of Biomedical Nanotechnology, vol. 4, no. 4, pp. 469-473, 2008.

[21] S. Bhattacharyya, R. A. Kudgus, R. Bhattacharya, and P. Mukherjee, "Inorganic nanoparticles in cancer therapy," Pharmaceutical Research, vol. 28, no. 2, pp. 237-259, 2011.

[22] S. J. McMahon, W. B. Hyland, M. F. Muir et al., "Biological consequences of nanoscale energy deposition near irradiated heavy atom nanoparticles," Scientific Reports, vol. 1, article 18, 2011.

[23] M. K. K. Leung, J. C. L. Chow, B. D. Chithrani, M. J. G. Lee, B. Oms, and D. A. Jaffray, "Irradiation of gold nanoparticles by X-rays: Monte Carlo simulation of dose enhancements and the spatial properties of the secondary electrons production," Medical Physics, vol. 38, no. 2, pp. 624-631, 2011.

[24] A. Anshup, J. S. Venkataraman, C. Subramaniam et al., "Growth of gold nanoparticles in human cells," Langmuir, vol. 21, no. 25, pp. 11562-11567, 2005.
[25] S. Jain, J. A. Coulter, A. R. Hounsell et al., "Cell-Specific Radiosensitization by gold nanoparticles at megavoltage radiation energies," International Journal of Radiation Oncology Biology Physics, vol. 79, no. 2, pp. 531-539, 2011.

[26] M. Triesscheijn, P. Baas, J. H. M. Schellens, and F. A. Stewart, "Photodynamic therapy in oncology," Oncologist, vol. 11, no. 9, pp. 1034-1044, 2006.

[27] D. K. Chatterjee and Z. Yong, "Upconverting nanoparticles as nanotransducers for photodynamic therapy in cancer cells," Nanomedicine, vol. 3, no. 1, pp. 73-82, 2008.

[28] R. R. Allison, V. S. Bagnato, and C. H. Sibata, "Future of oncologic photodynamic therapy," Future Oncology, vol. 6, no. 6, pp. 929-940, 2010.

[29] S. Yamaguchi, H. Kobayashi, T. Narita et al., "Sonodynamic therapy using water-dispersed $\mathrm{TiO}_{2}$-polyethylene glycol compound on glioma cells: comparison of cytotoxic mechanism with photodynamic therapy," Ultrasonics Sonochemistry, vol. 18 , no. 5, pp. 1197-1204, 2011.

[30] R. R. Allison, G. H. Downie, R. Cuenca, X.-H. Hu, C. J. H. Childs, and C. H. Sibata, "Photosensitizers in clinical PDT," Photodiagnosis and Photodynamic Therapy, vol. 1, no. 1, pp. 27-42, 2004.

[31] M. K. K. Oo, Multifunctional Gold Nanoparticles For Photodynamic Therapy of Cancer, Stevens Institute of Technology, Hoboken, NJ, USA, 2010.

[32] M. Kuruppuarachchi, H. Savoie, A. Lowry, C. Alonso, and R. W. Boyle, "Polyacrylamide nanoparticles as a delivery system in photodynamic therapy," Molecular Pharmaceutics, vol. 8, no. 3, pp. 920-931, 2011.

[33] P. Couleaud, V. Morosini, C. Frochot, S. Richeter, L. Raehm, and J.-O. Durand, "Silica-based nanoparticles for photodynamic therapy applications," Nanoscale, vol. 2, no. 7, pp. 10831095, 2010.

[34] M. Zeisser-Labouebe, N. Lange, R. Gurny, and F. Delie, "Hypericin-loaded nanoparticles for the photodynamic treatment of ovarian cancer," International Journal of Pharmaceutics, vol. 326, no. 1-2, pp. 174-181, 2006.

[35] E. Allemann, N. Brasseur, O. Benrezzak et al., "PEG-coated poly(lactic acid) nanoparticles for the delivery of hexadecafluoro zinc phthalocyanine to EMT-6 mouse mammary tumours," The Journal of Pharmacy and Pharmacology, vol. 47, no. 5, pp. 382-387, 1995.

[36] V. Saxena, M. Sadoqi, and J. Shao, "Polymeric nanoparticulate delivery system for indocyanine green: biodistribution in healthy mice," International Journal of Pharmaceutics, vol. 308, no. 1-2, pp. 200-204, 2006.

[37] E. Ricci-Junior and J. M. Marchetti, "Zinc(II) phthalocyanine loaded PLGA nanoparticles for photodynamic therapy use," International Journal of Pharmaceutics, vol. 310, no. 1-2, pp. 187-195, 2006.

[38] L. Liu, P. Miao, Y. Xu, Z. Tian, Z. Zou, and G. Li, "Study of $\mathrm{Pt} / \mathrm{Tio}_{2}$ nanocomposite for cancer-cell treatment," Journal of Photochemistry and Photobiology B, vol. 98, no. 3, pp. 207-210, 2010.

[39] S. Yamaguchi, H. Kobayashi, T. Narita et al., "Novel photodynamic therapy using water-dispersed $\mathrm{TiO}_{2}$ polyethylene glycol compound: evaluation of antitumor effect on glioma cells and spheroids in vitro," Photochemistry and Photobiology, vol. 86, no. 4, pp. 964-971, 2010.

[40] A. Janczyk, A. Wolnicka-Głubisz, K. Urbanska, H. Kisch, G. Stochel, and W. Macyk, "Photodynamic activity of platinum(IV) chloride surface-modified $\mathrm{TiO}_{2}$ irradiated with visible light," Free Radical Biology and Medicine, vol. 44, no. 6, pp. 1120-1130, 2008. 
[41] E. Fabian, R. Landsiedel, L. Ma-Hock, K. Wiench, W. Wohlleben, and B. van Ravenzwaay, "Tissue distribution and toxicity of intravenously administered titanium dioxide nanoparticles in rats," Archives of Toxicology, vol. 82, no. 3, pp. 151-157, 2008.

[42] B. K. Bernard, M. R. Osheroff, A. Hofmann, and J. H. Mennear, "Toxicology and carcinogenesis studies of dietary titanium dioxide-coated mica in male and female Fischer 344 rats," Journal of Toxicology and Environmental Health, vol. 29, no. 4, pp. 417-429, 1990.

[43] F. Bischoff and G. Bryson, "Tissue reaction to and fate of parenterally administered titanium dioxide. I. The intraperitoneal site in male Marsh-Buffalo mice," Research Communications in Chemical Pathology and Pharmacology, vol. 38, no. 2, pp. 279290, 1982.

[44] C. Yu, T. Canteenwala, M. E. El-Khouly et al., "Efficiency of singlet oxygen production from self-assembled nanospheres of molecular micelle-like photosensitizers $\mathrm{FC}_{4} \mathrm{~S}$," Journal of Materials Chemistry, vol. 15, no. 18, pp. 1857-1864, 2005.

[45] B. Ungun, R. K. Prud'homme, S. J. Budijono et al., "Nanofabricated upconversion nanoparticles for photodynamic therapy," Optics Express, vol. 17, no. 1, pp. 80-86, 2009.

[46] C. Guy and D. Ffytche, An Introduction to The Principles of Medical Imaging, Imperial College Press, London, UK, 2005.

[47] T. D. Schladt, K. Schneider, M. I. Shukoor et al., "Highly soluble multifunctional $\mathrm{MnO}$ nanoparticles for simultaneous optical and MRI imaging and cancer treatment using photodynamic therapy," Journal of Materials Chemistry, vol. 20, no. 38, pp. 8297-8304, 2010.

[48] W.-Y. Huang and J. J. Davis, "Multimodality and nanoparticles in medical imaging," Dalton Transactions, vol. 40, no. 23, pp. 6087-6103, 2011.

[49] E. B. Podgorsak, Radiation Oncology Physics: A Handbook for Teachers and Students, IAEA, Vienna, Austria, 2005.

[50] B.-J. Schultz, P. Wust, L. Ludemann, G. Jost, and H. Pietsch, "Monte Carlo simulation of contrast-enhanced whole brain radiotherapy on a CT scanner," Medical Physics, vol. 38, no. 8, pp. 4672-4680, 2011. 

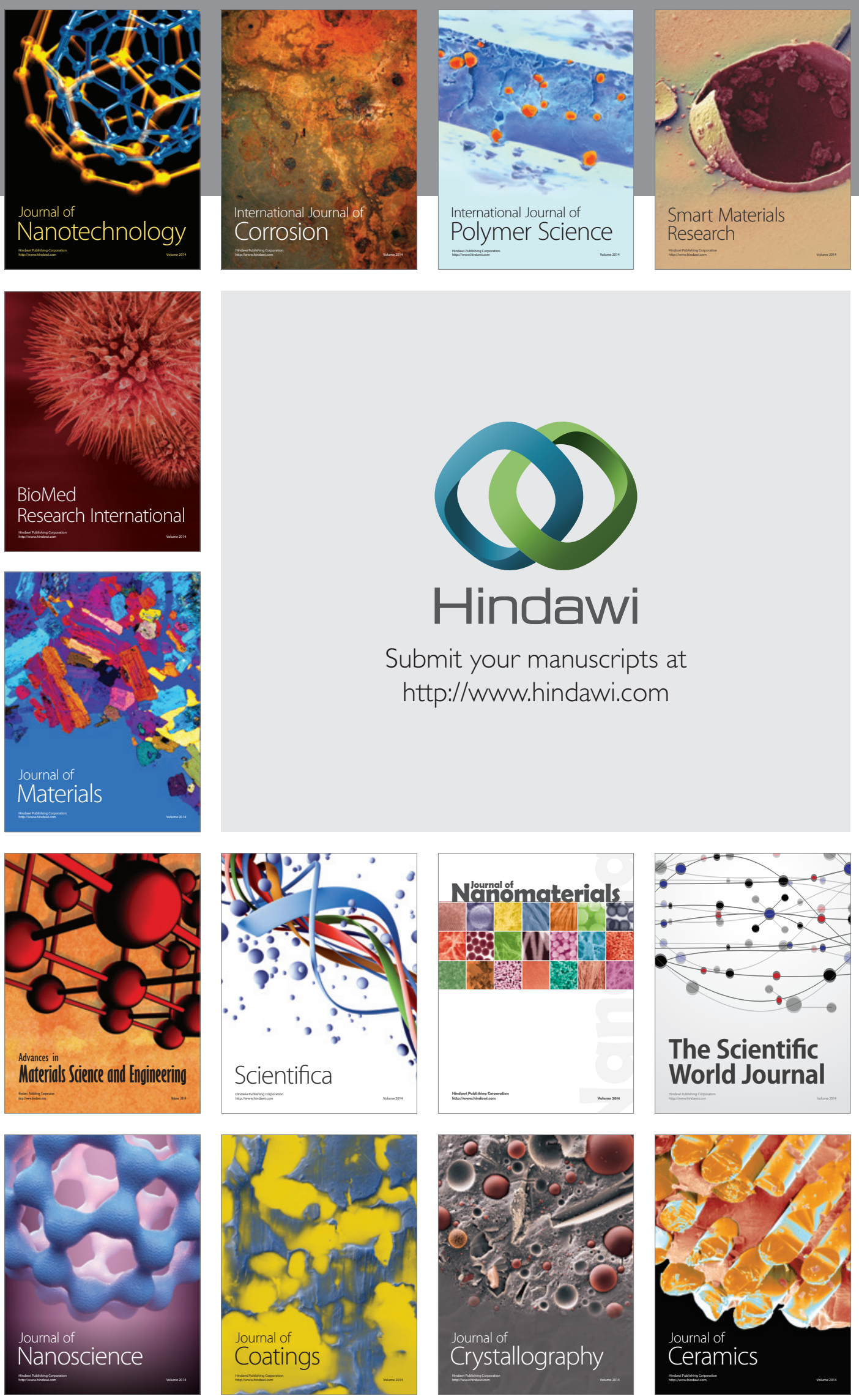

The Scientific World Journal

Submit your manuscripts at

http://www.hindawi.com

\section{World Journal}

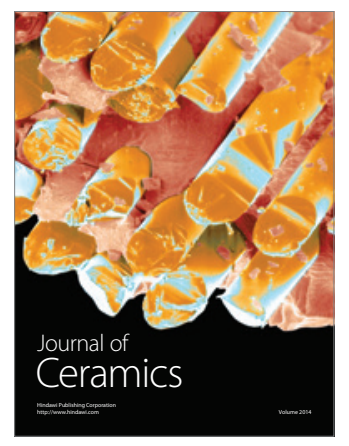

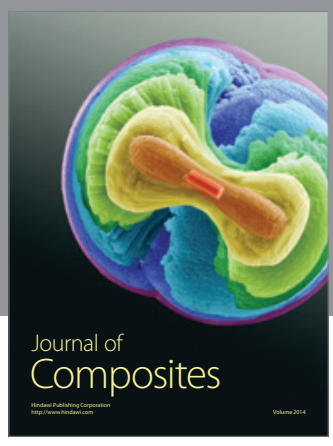
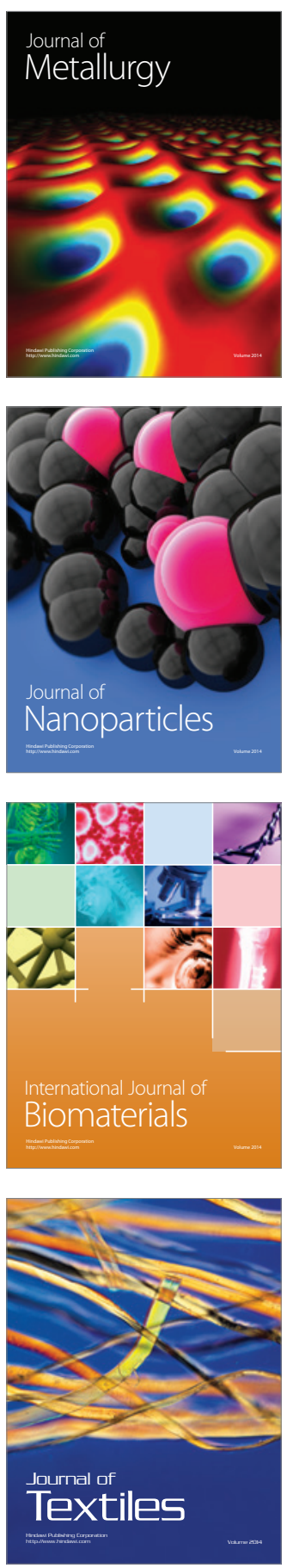